\title{
A importância do voluntariado para acadêmicos de enfermagem na vacinação contra a COVID-19: relato de experiência
}

The importance of volunteering for nursing students in vaccination against COVID-19: an experience report

La importancia del voluntariado para estudiantes de enfermería en vacunación contra COVID-19: un relato de experiencia

Rogéria de Sousa Rodrigues ${ }^{1 *}$, Jucilene Luz Neves${ }^{1}$, Juliana Conceição Dias Garcez ${ }^{1}$, Kyara Alessandra Mello Alves da Silva ${ }^{1}$, Maria Francilene da Silva Garcia ${ }^{1}$, Marina Pereira Queiroz dos Santos $^{1}$, Virginia Mercês Lara Pessoa Oliveira ${ }^{1}$, Dhessica Victoria Rodrigues de Souza ${ }^{1}$, Ana Carolina Marinho Pinheiro ${ }^{1}$, Karla Valéria Batista Lima².

\section{RESUMO}

Objetivo: Relatar a vivência de acadêmicos do curso de enfermagem experienciadas no decorrer do primeiro ano da campanha de vacinação de combate a COVID-19. Relato de experiência: Trata-se de um estudo do tipo relato de experiência, construído através da vivência adquirida pelos alunos de enfermagem que atuaram como voluntários na campanha de vacinação contra a COVID-19 em um posto de um Centro Universitário privado, o qual realizou uma parceria com a Secretaria Municipal de Saúde de Belém (SESMA), participaram como voluntários profissionais e alunos da área da saúde na campanha que se iniciou em 03/02/2021. Essa experiência levou os alunos a desenvolverem habilidades e competências tanto para a vida acadêmica quanto para a vida profissional. Considerações finais: Dessa forma, torna-se relevante o fortalecimento de incentivos a formação do acadêmico frente as necessidades de saúde pública, pois isto estimula um contato mais próximo a população, e possibilita um melhor desempenho.

Palavra-chave: Voluntariado, Acadêmicos, Enfermagem, COVID-19, Vacinação.

\section{ABSTRACT}

Objective: To report the experience of nursing students experienced during the first year of the vaccination campaign against COVID-19. Experience report: This is a study of the experience report type, built through the experience acquired by nursing students who acted as volunteers in the vaccination campaign against COVID-19 in a post of a private University Center, which they performed a partnership with the Municipal Health Department of Belém (SESMA), they participated as professional volunteers and students in the health area in the campaign that began on 02/03/2021. This experience led students to develop skills and competences for both academic and professional life. Final considerations: Thus, it is important to strengthen incentives for academic training in view of public health needs, as this encourages closer contact with the population, and enables better performance.

Keywords: Volunteering, Students, Nursing, COVID-19, Vaccination.

\section{RESUMEN}

Objetivo: Informar la experiencia vivida por estudiantes de enfermería durante el primer año de la campaña de vacunación contra COVID-19. Informe de experiencia: Se trata de un estudio del tipo informe de

${ }^{1}$ Centro Universitário Metropolitano da Amazônia (UNIFAMAZ), Belém - PA.

*E-mail: rogeriasousa440@yahoo.com.br

2 Instituto Evandro Chagas (IEC), Belém - PA. 
experiencia, construido a partir de la experiencia adquirida por estudiantes de enfermería que actuaron como voluntarios en la campaña de vacunación contra COVID-19 en un puesto de un Centro Universitario privado, que realizaron en alianza con la Municipalidad. Departamento de Salud de Belém (SESMA), participaron como voluntarios profesionales y estudiantes del área de salud en la campaña que inició el 02/03/2021. Esta experiencia llevó a los estudiantes a desarrollar habilidades y competencias tanto para la vida académica como profesional. Consideraciones finales: Por tanto, es importante fortalecer los incentivos a la formación académica ante las necesidades de salud pública, ya que esto favorece un contacto más estrecho con la población y permite un mejor desempeño.

Palabras clave: Voluntariado, Académicos, Enfermería, COVID-19, Vacunación.

\section{INTRODUÇÃO}

Desde o final de dezembro de 2019 a humanidade foi acometida por uma infecção causada pelo novo coronavírus, denominada SARS-COV-2, causador da doença COVID-19 (HERMIDA PMV, et al., 2020). Esse vírus foi detectado pela primeira vez na cidade de Wuhan na China, e possui como principal causa de mortalidade a Síndrome da Angústia Respiratória Aguda Grave (SRAG), podendo apresentar sintomas gripais comuns, com manifestações entre 5 e 6 dias após a infecção (BOTELHO JLS, et al., 2020).

Segundo dados epidemiológicos a transmissão ocorre diretamente de pessoa a pessoa pela via respiratória, através do contato próximo com a pessoa doente, ou seja, as gotículas são liberadas através da tosse, espirro e fala, ou indiretamente através de gotículas de aerossóis contidas em superfícies ou objetos com secreções contaminadas. Sendo altamente contagiosa entre os humanos, tornando-se uma pandemia devido ao seu elevado grau de transmissibilidade (NASCIMENTO CBC, et al., 2020).

Diante disso, algumas medidas foram tomadas de combate ao SARS-COV-2, como protocolos, medidas restritivas e etiquetas respiratórias, também foi desenvolvido o Plano Nacional de Operacionalização da Vacinação contra a COVID-19, uma medida adicional de resposta ao enfrentamento da doença devido a sua importância como emergência de saúde pública mundial e mediante ações de vacinação nos três níveis de gerenciamento (MINISTÉRIO DA SAÚDE, 2021a).

Frente os impactos causados pela pandemia, houve a mobilização entre diversos países, indústrias farmacêuticas e empresas que compartilhavam do mesmo interesse com intuito de fortalecer a produção de insumos para o desenvolvimento da vacina, assim como, houve muitos estudos que viabilizassem e garantissem a eficácia da vacina contra a COVID-19, tão logo houve em alguns países a liberação de algumas vacinas para uso emergencial todas com seu devido monitoramento para comprovação de sua eficácia e benefícios para a população (MINISTÉRIO DA SAÚDE, 2021a).

Segundo a Organização Mundial de Saúde (OMS), as vacinas passam por testes clínicos e fases específicas para terem sua segurança e eficácia comprovadas e liberadas para uso. Diante destas etapas de pesquisas, muitas vacinas foram candidatas e inicialmente poucas selecionadas para integrar o Programa Nacional de Imunização (PNI); sendo a CoronaVac, produzida pelo laboratório Sinovac/Butantã, a AstraZeneca proveniente da Universidade de Oxford com Fundação Osvaldo Cruz (Fiocruz), a vacina Pfizer/BioNTech, resultado de uma parceria americana e alemã. Assim como outras que ainda perpassam pelas fases de ensaios clínicos e fases de testes para posteriormente, se eficazes, serem liberadas (DOMINGUES CMAS, 2021).

Conforme o PNI a vacina é benéfica e seu uso é seguro, bem como suas estratégias de proteção à população, percebe-se que uma ampla cobertura vacinal, minimiza os riscos de agravos e adoecimentos da população, uma vez que reduz a propagação de agentes infecciosos, contribuindo com a saúde, proteção e maior qualidade de vida dos indivíduos não vacinados. Portanto, as vacinas foram liberadas após vários testes e estudos comprobatórios de sua eficácia, sendo enviada para vários países, dando início a uma campanha de proporção mundial para o combate ao novo coronavírus (LIMA EJF, et al., 2020). 
Porém, devido à limitação de vacinas para a completa cobertura vacinal, foram priorizados grupos específicos para serem imunizados, como aqueles que se encontravam em situações de riscos, por serem idosos ou mesmo por fazerem parte dos grupos que atuam na linha de frente no combate ao novo coronavírus (PEDREIRA NP, et al., 2021).

Nesse contexto, a vacina contra a COVID-19 iniciou no Brasil e no estado do Pará em janeiro de 2020, com a AstraZaneca e Coronavac, priorizando os profissionais de saúde, quilombolas e idosos acima de 90 anos, logo o calendário vacinal foi avançando na região metropolitana do estado e as idades foram reduzindo de acordo com a disponibilidade de vacinas (PEDREIRA NP, et al., 2021).

Assim, cabe ressaltar que o enfermeiro cumpre papel fundamental diante da vacinação da COVID-19, uma vez que dentre outras contribuições atua no planejamento, organização e execução das campanhas de vacinação, supervisionando as ações da equipe, monitorando os recursos materiais contribuindo para uma cobertura vacinal efetiva, direcionada ao alcance das metas planejadas frente à população (SOUZA JB, et al., 2021).

Ressalta-se a importância da enfermagem e sua ampla atuação em diversas áreas, destaca-se sua competência profissional diante da organização e dos cuidados prestados à população desde a atenção primária à alta complexidade, visto que a enfermagem é ativa e exerce ações de educação em saúde com foco na promoção, prevenção e reabilitação do ser humano. Logo, cumpre um papel fundamental que é desenvolver o cuidado com excelência, assim como, contribuir com a minimização dos riscos de morbidades a que os indivíduos estão expostos principalmente aqueles imunopreviníveis (LIMA EJF, et al., 2020).

Sendo assim, este trabalho teve por objetivo relatar a vivência de acadêmicos de enfermagem experienciadas no decorrer da campanha de vacinação de combate a COVID-19.

\section{RELATO DE EXPERIÊNCIA}

O presente estudo trata-se de um relato de experiência, construído através da vivência adquirida pelos alunos que atuaram como voluntários na campanha de vacinação contra a COVID-19 no posto de vacinação de um Centro Universitário privado, o qual realizou uma parceria com a Secretaria Municipal de Saúde de Belém (SESMA) em 2021, levando profissionais e alunos da área da saúde a serem voluntários na campanha que se iniciou em 03/02/2021. Tal experiência permitiu aos alunos o desenvolvimento de habilidades e competências tanto para a vida acadêmica quanto para a vida profissional, além do desenvolvimento de vários aspectos como liderança, trabalho em grupo, empatia, capacidade em resolver problemas dentre outros.

Segundo o Plano Nacional de Operacionalização da Vacinação Contra a COVID-19, foram priorizados alguns grupos para serem vacinados devido à alta suscetibilidade de infecção pelo SARS-CoV-2, esses grupos incluem: pessoas a partir de 60 anos institucionalizadas ou não, pessoas com deficiência institucionalizadas ou não, povos indígenas, trabalhadores da saúde, povos e comunidades tradicionais ribeirinhas, quilombolas, pessoas com comorbidades, gestantes e puérperas com comorbidades ou não, pessoas em situação de rua, funcionários do sistema de privação de liberdade, população privada de liberdade, trabalhadores da educação e dentre outros posteriormente incluídos (MINISTÉRIO DA SAÚDE, 2021b).

Na campanha de vacinação no referido Centro Universitário a equipe de voluntários realizou o desenvolvimento de funções específicas (triagem; registradores; vacinadores; apoio e supervisão) para melhor atender os usuários, e manter a organização e a qualidade do atendimento, como o treinamento de capacitação para as funções.

A equipe de triagem realizava o primeiro contato com o usuário e tinha como responsabilidade organizar as filas em ordem, em seguida a solicitação de documentos necessários como CPF, identidade, cartão do SUS e comprovante de residência, necessários para o registro da vacina, bem como realizar orientações e fornecer informações adequadas para o usuário sobre o grupo a ser vacinado, a impossibilidade de vacinarse caso estivesse com sintomas gripais ou tenha tomado outra vacina nos últimos 15 dias. 
A equipe de registro, obtinha uma responsabilidade muito importante nesse processo, pois identificavam se a pessoa já tomou ou não vacina em outro grupo prioritário, realizavam a validação da documentação, além de confeccionar a carteira de vacinação com todas as informações assim como o aprazamento da $2^{2}$ dose, por exemplo, além de realizarem o registro da pessoa no aplicativo desenvolvido pela prefeitura contendo: o tipo de vacina, o grupo prioritário, informando a data e o vacinador.

Já os vacinadores, poderiam ser enfermeiros ou técnicos de enfermagem com a carteira do Conselho Regional de Enfermagem (CORENB) ativo. Estes, cumpriam o papel final do processo, a administração da vacina. Portanto, mesmo sendo profissionais, estes, recebiam indispensavelmente um treinamento para a aspiração e técnica de aplicação das vacinas dadas as especificidades destas. Precisavam conhecer a organização das redes de frio, o porte da unidade para o adequado armazenamento dos imunobiológicos, assim como monitorar e controlar a temperatura entre $2^{\circ} \mathrm{C}$ a $8^{\circ}$ Celsius das caixas térmicas, mantendo a estabilidade e eficácia das vacinas, bem como estar atento para o tipo de vacina e o volume a ser administrado, informando ao público sobre os possíveis efeitos colaterais e a conduta adequada.

Ademais, o grupo de apoio realizava suporte aos demais membros da equipe, como a organização das filas, transporte de caixas térmicas, organização da alimentação e insumos da equipe.

Por fim, a supervisão, tratava-se de uma equipe responsável por uma série de atividades que eram desenvolvidas desde a organização da alimentação dos voluntários até a fiscalização do funcionamento da triagem, do registro, da aspiração bem como da administração dos imunizantes e esclarecendo de qualquer dúvida relacionada a estes processos. Além disso, essa equipe era responsável pela fiscalização e manutenção da temperatura dos imunobiológicos e do próprio ambiente. Eles realizavam também, a previsão, provisão e manutenção dos insumos como os Equipamentos de Proteção Individual (EPI) como seringas, agulhas, algodão, álcool etc. Também, tratava-se de uma equipe que representava o elo entre a instituição e a secretaria de saúde responsáveis pela resolução de problemas que surgissem no decorrer das atividades.

Todo este processo foi acompanhado por um profissional da SESMA que é o responsável pela supervisão, bem como para resolução de problemas que estivessem relacionados a secretaria de saúde. A campanha de vacina foi realizada dentro da instituição, que ofereceu suporte de infraestrutura como salas, auditórios climatizados, cadeiras para a organização da fila de espera, além de serviços de segurança para garantir a proteção dos profissionais e da população.

A rotina iniciava-se as $8 \mathrm{~h}$ manhã com orientações sobre o tipo de vacina, o lote, e o grupo prioritário a ser vacinado, após as informações repassadas a equipe era liberada, assim como a entrada das pessoas, que já munidos de senha eram direcionados a sala de espera na qual era feita a triagem e o registro, em seguida após realização das etapas anteriores direcionam-se a sala de administração do imunobiológico.

\section{DISCUSSÃO}

O Brasil é considerado um dos países mais comprometidos com a imunização, conquista essa afirmada desde a implantação do programa nacional de imunização (PNI) em 1973, o trabalho se dá com a total participação da enfermagem, a qual atualmente é separada por categorias (MONEZI NS e MARQUES D, 2019).

O PNI é uma referência internacional quando se trata de controle, eliminação e prevenção de doenças Imunopreveníveis, este sucesso se deve a segurança dos imunobiológicos, assim como da execução das recomendações a respeito da conservação, manipulação, administração e acompanhamento pós vacinal, tarefas essas realizadas pela equipe de enfermagem, onde o enfermeiro é o responsável pela supervisão das atividades na sala de vacina, assim como da educação permanente da equipe a qual pode ser composta por técnicos e auxiliares de enfermagem (RIBEIRO AB, et al., 2017).

Obedecendo ao protocolo a equipe de enfermagem tem funções específicas tais como administração dos imunizantes, registro das doses administradas, realizar aprazamentos para as próximas doses, manter a temperatura para conservação das vacinas, manter o controle dos lotes e da validade desses, bem como a orientação sobre as possíveis reações adversas pós vacina (CUNHA AG, et al., 2021). 
Logo, em meio a pandemia ocasionada pelo COVID-19 é inegável a importância desse processo de vacinação para a redução da incidência assim como de casos graves e óbitos, promovendo assim, saúde e proteção coletiva. Durante este processo é possível observar as diversas atividades que o enfermeiro desempenha no processo de prevenção e promoção à saúde, muitas delas não estão inclusas na sua bagagem teórica, questões essas que vão desde a logística, assim como o desenvolvimento de estratégias de assistência, do monitoramento dos indicadores bem como das reações adversas (BOSSE BR, et al., 2021).

Dentre as atividades realizadas pelo enfermeiro a etapa de registro é uma peça fundamental, pois nas posteriores análises das taxas de imunização, dados incorretos podem dificultar tais análise e impossibilitar a avaliação das metas estabelecidas pelos planos estaduais para a cobertura vacinal de determinados grupos (SÁ GS, et al., 2017).

Nesse sentido, o profissional de saúde quando munido de conhecimentos adequados faz a campanha vacinal ter grandes resultados, visto que é a partir de suas orientações que o usuário ganha confiança e adquire autonomia para tomar decisão acerca do seu processo de saúde-doença (CERQUEIRA ITA e BARBARA JS, 2016).

Entretanto, o estudo de Neto M, et al. (2020), relata que o contexto da pandemia visto pelas mídias sociais evidenciou o largo alcance da internet, em virtude disso muitas notícias são compartilhadas e criam uma rede de conteúdos com falsas informações conhecidas como Fake News, as mesmas são compartilhadas de forma rápida e instantânea, este fenômeno pode ser entendido como um vírus da comunicação e incita comportamentos contrário as orientações das autoridades de saúde.

Em consonância com o estudo de Moreira MRC, et al. (2021), é ressaltado que as notícias falsas se configuram como uma ameaça, fato esses já constatados em um estudo anterior a pandemia do Covid-19, segundo este estudo o desenvolvimento bem como aplicação das vacinas contra o Covid-19 alimentaram a disseminação de Fake News com vídeos e textos envolvendo desde a fase de testes clínicos até a aplicação dos imunobiológicos, incentivando assim a desconfiança quanto a composição e mecanismo de ação dos imunizantes, declarando que as vacinas poderiam causar mortes, alterar o material genético das pessoas ou implantar aparatos tecnológicos de monitoramento.

Nesse contexto, as práticas coletivas se constituem como um dos principais eixos para a formação dos profissionais de saúde acerca das demandas de saúde, exigências sociais, prestação de serviço à comunidade e disseminação de informações verídicas e baseadas em evidências. Dessa forma, a participação do acadêmico de enfermagem na campanha de vacinação contra a COVID-19 permite a ampliação de saberes, além do desenvolvimento de conhecimentos sobre autogestão, cooperação e saberes assistenciais (BOSSmE BR, et al., 2021).

Desse modo, a formação universitária deste profissional deve estar alicerçada no tripé ensino, pesquisa, extensão objetivando uma formação científica, profissional e cidadã de seus discentes, pois quando a formação se estende a atividades com a comunidade o universitário entende as demandas sociais emergentes e a realidade entre a teoria e a prática. E estendendo esse conceito a campanha de vacinação o voluntariado adquire para além de experiências assistenciais como o conhecimento sobre imunização, vacinas, aspiração e aplicação, este tem a chance de entender a logística de imunização de seu município (CAVALCANTE EEB, et al., 2019).

Além do mais, o acadêmico ainda garante a aquisição de uma nova experiência visto que, a humanidade vive uma pandemia e ter o papel de promover saúde é enriquecedor para o profissional em formação, pois permite aperfeiçoamento do saber-fazer do profissional (VASCONCELOS IA, et al., 2020; PEDREIRA NP, et al., 2021).

Dado o exposto ressalta-se a importância do voluntariado durante a campanha de vacinação contra a COVID-19, visto que, contribuiu significativamente para a formação profissional do acadêmico de enfermagem, além, de fortalecer as ações de educação em saúde, medida que possibilitou maior segurança aos acadêmicos diante de novas experiências adquiridas frente a situações de agravos da COVID-19. Dessa forma, torna-se relevante o fortalecimento de incentivos a formação do acadêmico frente as necessidades de saúde pública, pois isto estimula um contato mais próximo a população, e possibilita um melhor desempenho. 


\section{REFERÊNCIAS}

1. BOTELHO JLS, et al. Campanha de vacinação na pandemia de sars cov 2: Relato de experiência. Revista nursing, $2021 ; 24(272): 5092-5094$.

2. BOSSE BR, et al. Campanha de vacinação COVID-19 em Santa Maria, Rio Grande do Sul: relato de experiência. Congresso internacional em saúde, 2021.

3. CAVALCANTE EEB, et al. As atividades de extensão acadêmica conforme o ordenamento jurídico vigente: um estudo de caso sobre a Universidade Federal de Rondônia. Revista Interdisciplinares do Direito, 2019; 1(1).

4. CERQUEIRA ITA, BARBARA JS. Atuação da enfermeira na sala de vacinação em unidades de saúde da família. Revista Baiana de Saúde Pública, 2016; 40(2): 442-256.

5. CUNHA AG, et al. Atuação da enfermagem na campanha de vacinação contra a COVID-19 em um Centro Universitário em Belém-PA. Research, Society and Development, 2021; 10(8): e35310816835.

6. COLICHI RMB, et al. O papel do enfermeiro de atenção primária em saúde na vigilância epidemiológica: reflexões para pandemia de COVID-19. Revista Saúde Coletiva avanços e desafios para a integralidade do cuidado, 2021.

7. DOMINGUES CMAS. Desafios para a realização da campanha de vacinação contra a COVID-19 no Brasil. Cad. Saúde Pública, 2021; 37(1).

8. NASCIMENTO CBC, et al. SARS-CoV2 e Covid-19: aspectos fisiopatológicos e imunológicos, estratégias de diagnóstico e desenvolvimento de vacinas. Revista Interdisciplinar de Saúde e Educação, 2020; 1(2): $122-158$.

9. HERMINIA PMV, et al. Cuidados à pessoa suspeita de covid-19 com sinais de gravidade na atenção primária à saúde. Enfermagem em Foco. 2020; 30: 192-198.

10. LIMA EJF, et al. Vacinas para COVID-19: perspectivas e desafios. Revista Residência Pediátrica 2020; 10(2-04).

11. MINISTÉRIO DA SAÚDE. Secretaria extraordinária de enfrentamento à covid-19. Plano nacional de operacionalização da vacinação contra a covid-19. $11^{\text {a }}$ ed. 2021a. Disponível em: https://www.gov.br/saude/ptbr/coronavirus/publicacoes-tecnicas/guias-e-planos/plano-nacional-de-vacinacao-covid-19/view. Acesso em: 09 de setembro de 2021.

12. MINISTÉRIO DA SAÚDE. Secretaria de vigilância em saúde Plano nacional de operacionalização da vacinação contra a covid-19. 5 a $\quad$ ed. 2021b. Disponível em: https://www.gov.br/saude/pt br/media/pdf/2020/dezembro/16/plano_vacinacao_versao_eletronica-1.pdf. Acesso em: 09 de setembro de 2021.

13. MOREIRA MRC, et al. Categorias das fake news sobre COVID-19 disseminadas no primeiro ano da pandemia no Brasil. Mundo da Saúde 2021,45: 221-232.

14. MONEZI NS, MARQUES D. Práticas de Enfermagem nas salas de vacina da Atenção Básica. Revista dos Trabalhos de Iniciação Científica da UNICAMP, Campinas, SP 2019; 27.

15. NETO M, et al. Fake News no cenário da pandemia de COVID-19. Cogitare Enferm. 2020; 25: e72627.

16. PEDREIRA NP, et al. Vivência do acadêmico de enfermagem frente à campanha de vacinação ao combate a pandemia da COVID-19. Revista Eletrônica Acervo Saúde, 2021; 13(5).

17. RIBEIRO $A B$, et al. A importância da atuação do enfermeiro na sala de vacina: uma revisão integrativa. R. Enferm. UFJF - Juiz de Fora, 2017; 3(1): 37- 44.

18. SÁ GS, et al. Registro correto de vacinas: capacitação para equipe de enfermagem. Anais do EVINCI-UniBrasil, 2017; 3(1): 174-174.

19. SOUZA JBD, et al. Campanha de vacinação contra COVID-19: diálogos com enfermeiros atuantes na Atenção Primária à Saúde. Revista da Escola de Enfermagem da USP, 2021; 55.

20. SOUZA TS, et al. Mídias Sociais e educação em saúde: o combate às Fakes News na pandemia pela COVID-19. Enferm. Foco, 2020; 11(1): 124-130.

21. VASCONCELOS IA, et al. Imunização frente ao contexto do Covid-19. Research, Society and Development, 2020. 